NUB-3262

YITP-SB-08-50

\title{
PAMELA Positron Excess as a Signal from the Hidden Sector
}

\author{
Daniel Feldman ${ }^{1}$, Zuowei $\mathrm{Liu}^{2}$, and Pran Nath ${ }^{1}$ \\ ${ }^{1}$ Department of Physics, Northeastern University, Boston, MA 02115, USA, \\ ${ }^{2}$ C. N. Yang Institute for Theoretical Physics, \\ Stony Brook University, Stony Brook, NY 11794, USA. \\ V1 Dated: Oct 31, 2008.
}

\begin{abstract}
The recent positron excess observed in the PAMELA satellite experiment strengthens previous experimental findings. We give here an analysis of this excess in the framework of the Stueckelberg extension of the standard model which includes an extra $U(1)_{X}$ gauge field and matter in the hidden sector. Such matter can produce the right amount of dark matter consistent with the WMAP constraints. Assuming the hidden sector matter to be Dirac fermions it is shown that their annihilation can produce the positron excess with the right positron energy dependence seen in the HEAT, AMS and the PAMELA experiments. The predictions of the $\bar{p} / p$ flux ratio also fit the data.
\end{abstract}


Introduction: An excess of the positron flux emanating from the galactic halo has been reported by the PAMELA satellite experiment[1] which supports the previous observations by the HEAT and AMS experiments [2] but is much more accurate. A remarkable feature of the positron spectrum is the turn around and increase in the positron flux with positron energy in the range of $10-80 \mathrm{GeV}$. Here we analyze the possibility that the positron flux is arising from the annihilation of particles in the hidden sector. The hidden sector, which is defined as a sector with fields which are neutral under the standard model (SM) gauge group, has played an increasingly important role since its inception in the formulation of supergravity grand unification[3]. Subsequently, it was realized that such hidden sectors appear quite naturally in the context of string theory[4]. More recently the hidden sector was utilized in the Stueckelberg extension of the standard model. In such an extension, the SM gauge group $S U(3)_{C} \times S U(2)_{L} \times U(1)_{Y}$ is supplemented by an extra $U(1)_{X}$ gauge group factor [5, 6] where the SM fields are neutral under the $U(1)_{X}$. However, the mixings between the SM and the hidden sector do occur via a connector sector which mixes the gauge fields of $U(1)_{Y}$ and of $U(1)_{X}$. Such mixings can occur via the Stueckelberg mass terms[5] or via kinetic mixings|7] or both[8]. Electroweak constraints from the LEP and the Tevatron were analyzed in [5, 6, 8] and an analysis of the dark matter was given with and without kinetic mixing in [8, 9, 10] consistent with the WMAP [11] constraints. (For related works on the $U(1)$ extensions see [7, 12], and for other works on the hidden sector see [13]). Further recent works regarding Stueckelberg extensions in the context of the string and D brane models can be found in [14] and for related works see [15]. We note that hidden sectors are also central to unparticle[16] and ungravity models [17].

Positron fraction from annihilation in the hidden sector: The analysis of the positron spectrum depends both on the particle physics as well as on the astrophysical models and these features have been discussed recently in some detail in [18, 19]. Here we focus on the fit to the just released data by the PAMELA experiment[1] from annihilation of dark matter in the hidden sector in the framework of Stueckelberg extension of the standard model. We give now the details of the analysis.

In general the positron flux arising from the annihilation of dark matter (DM) particles is given by [20, 21]

$$
\Phi_{e^{+}}=\frac{\eta B v_{e^{+}}}{4 \pi b(E)} \frac{\rho^{2}}{M_{D}^{2}} \int_{E}^{M_{D}} \sum_{k}\langle\sigma v\rangle_{k H}\left(\frac{d N_{e^{+}}}{d E^{\prime}}\right)_{k} \mathcal{I}_{\left(E, E^{\prime}\right)} d E^{\prime}
$$


where $M_{D}$ is the mass of the dark matter particle, $\eta=1 / 2(1 / 4)$ for the DM particle being Majorana or Dirac [20], $\mathrm{B}$ is the boost factor which is expected to lie in the range (2-10) although significantly larger values have been used in the literature. In the above $v_{e^{+}}$is the positron velocity where $v_{e^{+}} \sim c$, and $\rho$ is the local dark matter density in the halo so that $\rho$ lies in the range $(0.2-0.7)\left[\mathrm{GeV} / \mathrm{cm}^{3}\right]$ [22]. Further, $b(E)$ in Eq.(11) is given by [23, 24, 25] $b(E)=E_{0}\left(E / E_{0}\right)^{2} / \tau_{E}$, where $\tau_{E} \sim 10^{16}[\mathrm{~s}]$, with $E$ in $[\mathrm{GeV}]$ and $E_{0} \equiv 1 \mathrm{GeV}$. Here $\langle\sigma v\rangle_{H}$ is the velocity averaged cross section in the Halo $(H)$ of the galaxy as emphasized by the subscript $H$. In some works $\langle\sigma v\rangle_{H}$ is replaced by the $\langle\sigma v\rangle_{X_{f}}$ at the freezeout temperature. However, such an approximation can lead to significant errors since the ratio $\langle\sigma v\rangle_{H} /\langle\sigma v\rangle_{X_{f}}$ can deviate significantly from unity depending on the part of the parameter space one is in. The halo function $\mathcal{I}_{\left(E, E^{\prime}\right)}$ is parametrized as in [21], and we consider both the Navarro, Frenk and White (NFW) and Moore et. al [26] profiles coupled with various diffusion models.

The channels that can contribute to the positron flux in the model are $D \bar{D} \rightarrow$ $f \bar{f}, W^{+} W^{-}, \ldots$ where $f$ is any quark or lepton final state of the standard model. In our analysis we make use of the dominance of the direct channel poles, specifically of the $Z^{\prime}$ pole, and here the $W^{+} W^{-}$final state contribution is much suppressed relative to the $f \bar{f}$ final state contribution[6]. We begin by discussing the $D \bar{D} \rightarrow f \bar{f}$ cross section in Stueckelberg extension of the standard model . Here one has a mixed Higgs mechanism and a Stueckelberg mechanism to break the extended $S U(2)_{L} \times U(1)_{Y} \times U(1)_{X}$ electroweak symmetry [5]. After such breaking there is the usual mixing between the neutral component of the $S U(2)_{L}$ gauge field $A_{3}^{\mu}$ and the $U(1)_{Y}$ gauge field $B^{\mu}$. Thus together the Higgs mechanism and the Stueckelberg mechanism generate a $3 \times 3$ mass matrix for the three gauge fields $C^{\mu}, B^{\mu}, A_{3}^{\mu}$. The above model has a non-diagonal mass matrix $\left(M_{\mathrm{St}}^{2}\right)[5]$ and a non-diagonal kinetic mixing matrix $(\mathcal{K})[7$, 8]. A diagonalization of the kinetic term can be obtained by a GL(3) transformation. However, such a diagonalization is arbitrary up to an orthogonal transformation. One can choose a combination $K^{\prime}$ of $\mathrm{GL}(3)$ and an orthogonal transformation such that $\mathcal{M}^{2}=K^{\prime T} M_{S t}^{2} K^{\prime}$ has a form similar to that of $M_{S t}^{2}$, i.e., the zero elements of $M_{S t}^{2}$ are maintained by the transformation (for details see [8]). In the basis where both the kinetic and the mass $^{2}$ matrix are diagonal one has $\mathcal{M}_{\text {Diag }}^{2}=\mathcal{R}^{T} \mathcal{M}^{2} \mathcal{R}$. In this basis the interactions between the standard model fermions and the vector bosons $Z, Z^{\prime}$ are given by $\mathcal{L}_{\text {int }}^{\mathrm{VS}}=\bar{f} \gamma^{\mu}\left[C_{f_{L}}^{Z^{\prime}} P_{L} Z_{\mu}^{\prime}+C_{f_{L}}^{Z} P_{L} Z_{\mu}+(L \rightarrow R)\right] f$, where as usual $Q=T_{L, R}^{3}+Y_{L, R} / 2, T_{R}^{3}=0, P_{L, R}=\frac{1}{2}\left(1 \mp \gamma^{5}\right)$, and $C_{f_{L}}^{Z_{i}}=T_{L}^{3}\left[g_{2} \mathcal{R}_{3 i}-\gamma \sqrt{1+\bar{\epsilon}^{2}} \mathcal{R}_{2 i}\right]$, 

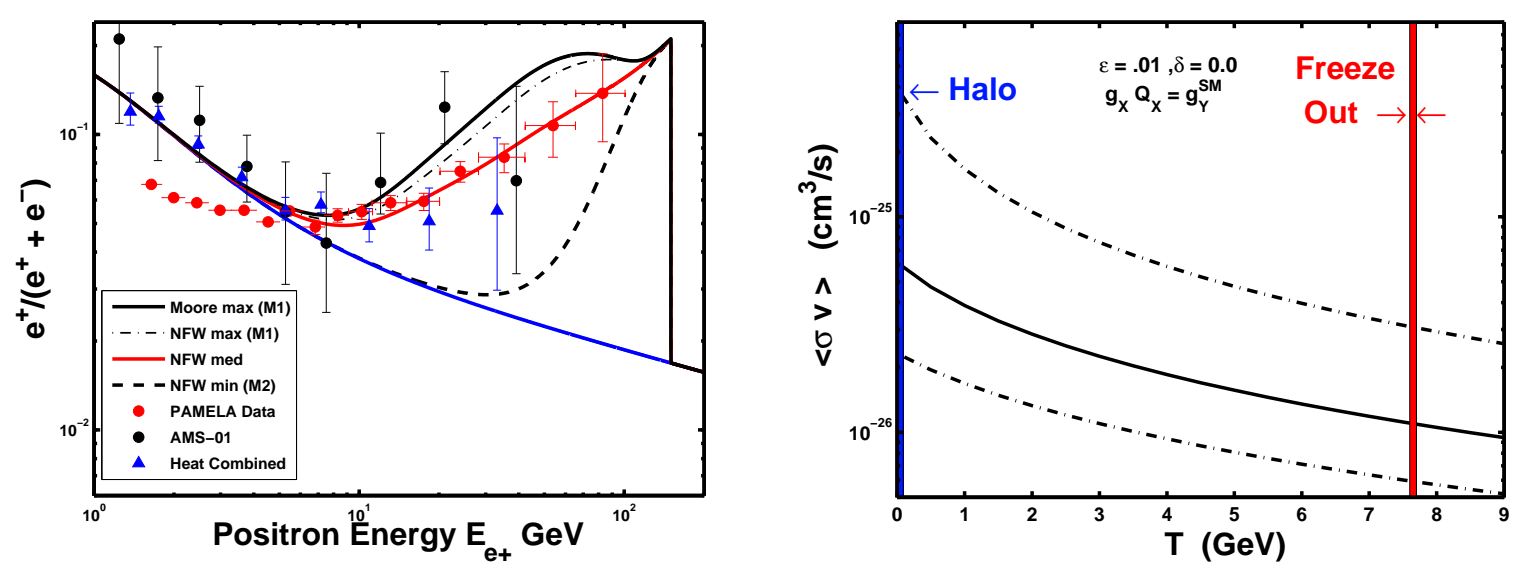

FIG. 1: (Left Panel): Positron spectrum including the monochromatic source and continuum flux for various halo/diffusion models with $(\epsilon=0.006, \delta=0.00)$ and $\rho=0.35 \mathrm{GeV} / \mathrm{cm}^{3}$ with $M_{Z^{\prime}}=298$ $\mathrm{GeV}, M_{D}=150 \mathrm{GeV}, \tau_{E}=3 \times 10^{16} \mathrm{~s}[23]$, and $\mathrm{B}=10$; and $\Omega h^{2}=0.13$ (calculated by integration over the Breit-Wigner pole). Also plotted is the just released PAMELA data [1], along with the AMS-01 and HEAT data 2]. The background flux ratio is the decaying solid (blue) lower curve. (Right Panel): An exhibition of the dependence of $\langle\sigma v\rangle$ on temperature for Stueckelberg models as given in the figure with $M_{D} / \mathrm{GeV} \in[150,153]$ in steps of 1.5 and $M_{Z^{\prime}}$ fixed as in the left panel of Fig(1). The annihilation near a pole generates a significant enhancement of $\langle\sigma v\rangle_{H}$ in the halo relative to $\langle\sigma v\rangle_{X_{f}}$ at freezeout. The natural Breit-Wigner enhancement of $\langle\sigma v\rangle_{H}$ obviates the necessity of using very large boost factors.

$C_{f_{R}}^{Z_{i}}=Q \gamma \sqrt{1+\bar{\epsilon}^{2}} \mathcal{R}_{2 i}$, where $Z_{i}=\left(Z^{\prime}, Z\right)$ for $i=1,2$. The photon couples as usual to the visible fermionic fields with strength $e Q$, and $\gamma$ is related to $e$ by $e=\gamma g_{2} /\left[\gamma^{2}+g_{2}^{2}\right]^{1 / 2}$. The Dirac fermion is assumed to have the interaction $g_{X} Q_{X} \bar{D} \gamma^{\mu} D C_{\mu}$ which in the diagonal basis gives $\mathcal{L}_{\text {int }}^{\mathrm{HS}}=\bar{D} \gamma^{\mu}\left[C_{D}^{Z^{\prime}} Z_{\mu}^{\prime}+C_{D}^{Z} Z_{\mu}+C_{D}^{\gamma} A_{\mu}^{\gamma}\right] D$, where $C_{D}^{\gamma}=g_{X} Q_{X}\left(-c_{\theta} s_{\phi}-S_{\delta} c_{\theta} c_{\phi}\right), C_{D}^{Z}=$ $g_{X} Q_{X}\left(s_{\psi} c_{\phi}+s_{\theta} s_{\phi} c_{\psi}-S_{\delta}\left(s_{\psi} s_{\phi}-s_{\theta} c_{\phi} c_{\psi}\right)\right), C_{D}^{Z^{\prime}}=g_{X} Q_{X}\left(c_{\psi} c_{\phi}-s_{\theta} s_{\phi} s_{\psi}-S_{\delta}\left(c_{\psi} s_{\phi}+s_{\theta} c_{\phi} s_{\psi}\right)\right)$. Here $S_{\delta}=\delta / \sqrt{1-\delta^{2}}$ and the angles $\theta, \phi, \psi$ appear in the rotation matrix $\mathcal{R}$, and are defined by $\tan \theta=\gamma / g_{2}, \tan \phi=\bar{\epsilon}=[\epsilon-\delta]\left[1-\delta^{2}\right]^{-\frac{1}{2}}$ and $\psi$ is determined by the relation $\tan 2 \psi=2 \bar{\epsilon} M_{0} \sqrt{M_{0}^{2}-M_{W}^{2}} /\left(M_{1}^{2}-M_{0}^{2}+\bar{\epsilon}^{2}\left(M_{0}^{2}+M_{1}^{2}-M_{W}^{2}\right)\right)$, where $M_{0}=v \sqrt{g_{2}^{2}+\gamma^{2}} / 2$. The parameters $\epsilon$ and $\delta$ are constrained by the electroweak data $[6,8]$. One finds that $\epsilon$ and $\delta$ are both separately constrained so that $|\epsilon|,|\delta| \lesssim .06$. The action given in [5] leads to an 
integrated cross section[10],

$$
\sigma_{f \bar{f}} \simeq \frac{N_{f} s}{32 \pi} \frac{\beta_{f}}{\beta_{D}}\left[\left(\left|\xi_{L}\right|^{2}+\left|\xi_{R}\right|^{2}\right) \cdot F_{1}+\operatorname{Re}\left(\xi_{L}^{*} \xi_{R}\right) \cdot F_{2}\right]
$$

where $F_{1}=1+\beta_{D}^{2} \beta_{f}^{2} / 3+4 M_{D}^{2} s^{-1}\left(1-2 m_{f}^{2} / s\right)$, and $F_{2}=8 m_{f}^{2} s^{-1}\left(1+2 M_{D}^{2} / s\right)$. Here $\beta_{f, D}=\left(1-4 m_{f, D}^{2} / s\right)^{1 / 2}, s=4 m_{D}^{2} /\left(1-v^{2} / 4\right)$ and $\xi_{L, R}$ include the poles

$$
\xi_{L, R}=\frac{C_{D}^{\gamma} e Q}{s}+\frac{C_{D}^{Z} C_{f_{L, R}}^{Z}}{s-M_{Z}^{2}+i \Gamma_{Z} M_{Z}}+\frac{C_{D}^{Z^{\prime}} C_{f_{L, R}}^{Z^{\prime}}}{s-M_{Z^{\prime}}^{2}+i \Gamma_{Z^{\prime}} M_{Z^{\prime}}} .
$$

The dominant term in our analysis is the line source arising from the annihilation $D \bar{D} \rightarrow$ $Z^{\prime} \rightarrow e^{+} e^{-}$and in this case one has $\sum_{F=\text { Final states }}\langle\sigma v\rangle_{F}\left(d N_{e^{+}} / d E^{\prime}\right)_{F} \sim\langle\sigma v\rangle_{e^{+} e^{-}} \delta\left(E^{\prime}-\right.$ $\left.M_{D}\right)+\ldots$, where the dots stand for the background terms that contribute to the continuum flux. The continuum flux arises mostly from muons and to a much lesser degree from taus[28]. Defining $R_{f}$ as the positron ratio from source $f$ one finds $R_{\mu} / R_{e}$ is non-negligible and decreases with increasing $E_{e^{+}}$over the dark matter mass (DM) mass range of interest and a similar relation holds for the taus[28]. The inclusion of the flux from the continuum reduces the needed boost factor slightly, however the line source still dominates at high energies. The use of the above in Eq.(1) yields the primary positron flux $\Phi_{e^{+}} \equiv \Phi_{e^{+}}^{(1)}$. We must add to it the secondary positron flux $\Phi_{e^{+}}^{(2)}$ and then compare it with the electron flux $\left(\Phi_{e^{-}}^{(1)}+\Phi_{e^{-}}^{(2)}\right)$. For $\Phi_{e^{-}}^{(1)}, \Phi_{e^{-}}^{(2)}, \Phi_{e^{+}}^{(2)}$ we use the parametrizations of [27, 29]. For comparison with experiment one often defines the positron fraction $e^{+} /\left(e^{+}+e^{-}\right)$, and an analysis is given of this observable as a function of the positron energy in Fig.(1) for the Stueckelberg $Z^{\prime}$ model. One finds that the annihilation of Dirac fermions via the $Z^{\prime}$ pole into $e^{+} e^{-}+\mu^{+} \mu^{-}$gives a sufficient kick to generate the necessary turn around in the positron fraction at just about the desired value of the positron energy consistent with the relic density constraints. The analysis of Fig.(1) (left panel) exhibits the theoretical evaluation for several model points. Here we consider NFW min (M2), med and $\max$ (M1) as well as the Moore max (M1) parametrizations [20, 21]. One finds that there is a significant variation in the prediction depending on the profile/diffusion model one chooses. However, one finds that the PAMELA data does lie in the range of the theoretical predictions. We note in passing that the gamma ray spectrum in this model has been discussed in[10]. The theoretical predictions cover a range which includes the PAMELA data[1]. Further, such a fit determines the dark matter fermion mass to be roughly half the $Z^{\prime}$ mass. 


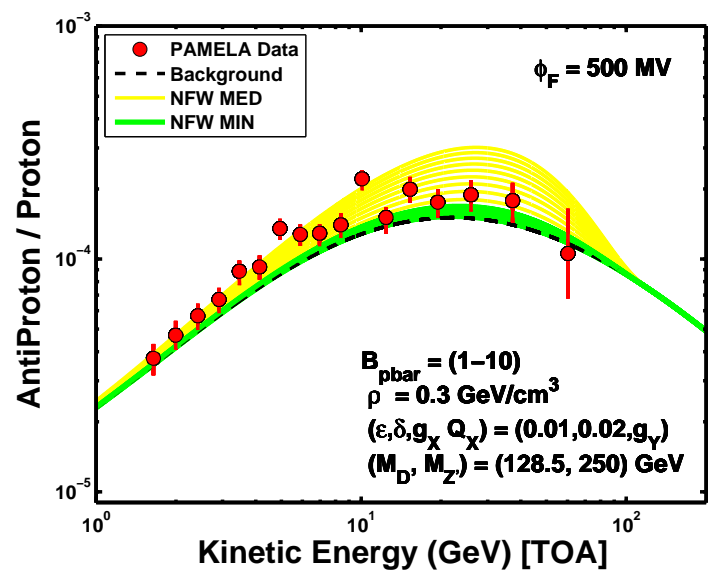

FIG. 2: The $\bar{p} / p$ flux ratio including the TOA correction to the IS spectrum [31], and with $B_{\bar{p}} \in$ $(1-10)$. The green (darker) curves (NFW min) are insensitive to the boost in the ratio, while the yellow (lighter) curves (NFW med) allow a boost as large as 5 or even larger.

In Fig.(11) (right panel) we exhibit the dependence of $\langle\sigma v\rangle$ on temperature. The analysis shows that $\langle\sigma v\rangle$ can have a significant temperature dependence. Thus the simplifying assumption often made in assuming that $\langle\sigma v\rangle$ is a constant as one moves from the freezeout temperature to the temperature of the galactic halo is erroneous. Specifically the analysis shows that the temperature dependence is model dependent and one can generate an enhancement of $\langle\sigma v\rangle_{H}$ in the halo relative to freezeout $\langle\sigma v\rangle_{X_{f}}$ by as much as a factor of 10 or more depending on the part of the parameter space one is in. Typically the temperature dependence is enhanced when the dark matter particles annihilate near a pole from the Breit-Wigner which is the case in the analysis here.

Anti-proton Flux and PAMELA : The $\bar{p} / p$ flux ratio as recently reported by the PAMELA[30] collaboration indicates a smooth increase with energy up to about $10 \mathrm{GeV}$ and then a flattening out in agreement with the background and with previous experiments. We note that a suppression of $\bar{p} / p$ flux ratio is possible in the model presented here. This is due in part because the $Z^{\prime} \rightarrow W^{+} W^{-}$is suppressed as already discussed. We have carried out a detailed analysis of the $\bar{p} / p$ flux ratio. Our analysis follows closely the work of [21] with fragmentation functions as modeled in Bottino et al and by Bergstrom etal and $(p, \bar{p})$ backgrounds as in Donato et al and Bringmann et al [31]. The Interstellar (IS) flux has been modified for predictions at the Top of the Atmosphere (TOA) which suffers from large uncertainties. The results are given in Fig.(2) and compared with the recently reported 

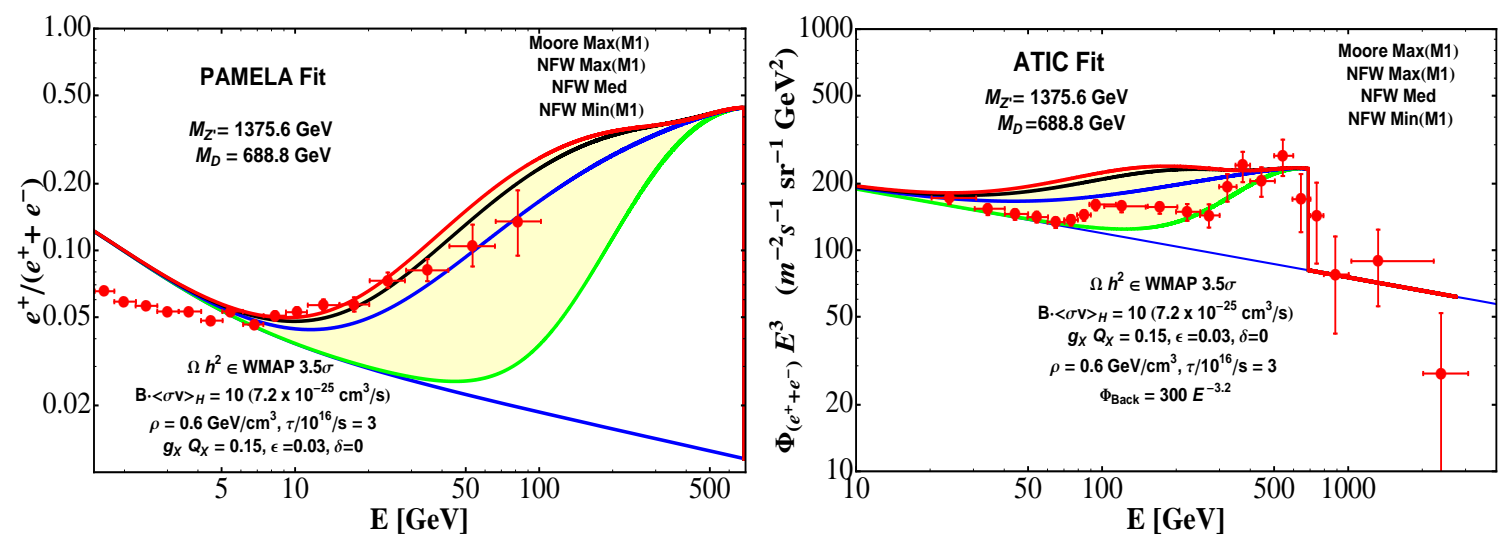

FIG. 3: Fit to the PAMELA and ATIC [32] data for a heavy Dirac dark matter mass of $688.8 \mathrm{GeV}$ with the Breit-Wigner enhancement. The curves in descending order are for the cases for the halo profiles listed on the top right hand corner.

results by the PAMELA collaboration. It is found that the $\bar{p} / p$ analysis of Fig.(2) is fully compatible with the recent PAMELA data. It is further observed that the NFW min profile, for the $\bar{p} / p$ predictions, are rather insensitive to a boost factor, while boost factors as large as 5 or larger are acceptable in the NFW med model. We note in passing that the $\bar{p} / p$ flux ratio does suffer from larger theoretical uncertainties than the $e^{+} / e$ flux ratio due to a larger diffusion length. Further, it is known that local inhomogeneities in the dark matter density may lead to very different boost factors for positrons and antiprotons (see, for example, Lavalle etal in [31]).

Conclusion: In this work we have shown that the annihilation of the Dirac fermions in the hidden sector close to the $Z^{\prime}$ pole can generate a positron fraction compatible with the current PAMELA data. Specifically the model produces the right amount of positron spectrum enhancement with increasing positron energy indicated by the AMS-01 and the HEAT data and confirmed by the PAMELA data, and additionally the model can accommodate the antiproton constraints. A further support of the model can come from a direct observation of the $Z^{\prime}$ boson at the Large Hadron Collider.

Note Added: After submission of this paper, the ATIC Collaboration published its data on the electron excess[32]. One can fit both PAMELA and ATIC as well as the $\bar{p} / p$ flux in the model with a change of the dark matter and $Z^{\prime}$ mass and with a low boost factor of 10. This fit is given in Fig.(3) and includes the continuum flux. Again the Breit-Wigner enhancement plays an important role in the analysis. 
Acknowledgments: This research is supported in part by NSF grants PHY-0653342 (Stony Brook) and PHY-0757959 (NU).

[1] O. Adriani et al., arXiv:0810.4995 [astro-ph] (Submitted to Nature).

[2] S. W. Barwick et al. [HEAT Collaboration], Astrophys. J. 482, L191 (1997); M. Aguilar et al. [AMS-01 Collaboration], Phys. Lett. B 646 (2007) 145.

[3] A. H. Chamseddine, R. L. Arnowitt and P. Nath, Phys. Rev. Lett. 49, 970 (1982); L. J. Hall, J. D. Lykken and S. Weinberg, Phys. Rev. D 27, 2359 (1983).

[4] P. Candelas, G. T. Horowitz, A. Strominger and E. Witten, Nucl. Phys. B 258, 46 (1985).

[5] B. Kors and P. Nath, Phys. Lett. B 586, 366 (2004) arXiv:hep-ph/0402047; JHEP 0412, 005 (2004) arXiv:hep-ph/0406167]; JHEP 0507, 069 (2005) arXiv:hep-ph/0503208.

[6] D. Feldman, Z. Liu and P. Nath, Phys. Rev. Lett. 97, 021801 (2006) arXiv:hep-ph/0603039; JHEP 0611, 007 (2006) arXiv:hep-ph/0606294.

[7] B. Holdom, Phys. Lett. B 166, 196 (1986), B 259, 329 (1991); K. S. Babu, C. F. Kolda and J. March-Russell, Phys. Rev. D 57, 6788 (1998).

[8] D. Feldman, Z. Liu and P. Nath, Phys. Rev. D 75, 115001 (2007) arXiv:hep-ph/0702123.

[9] D. Feldman, B. Kors and P. Nath, Phys. Rev. D 75, 023503 (2007) arXiv:hep-ph/0610133.

[10] K. Cheung and T. C. Yuan, JHEP 0703, 120 (2007) arXiv:hep-ph/0701107.

[11] D. N. Spergel et al. [WMAP Collaboration], Astrophys. J. Suppl. 170, 377 (2007).

[12] J. Kumar and J. D. Wells, Phys. Rev. D 74, 115017 (2006); W. F. Chang, J. N. Ng and J. M. S. Wu, Phys. Rev. D 74, 095005 (2006).

[13] T. Han, Z. Si, K. M. Zurek and M. J. Strassler, JHEP 0807, 008 (2008); Y. Gershtein, F. Petriello, S. Quackenbush and K. M. Zurek, arXiv:0809.2849 [hep-ph]. M. Ahlers, H. Gies, J. Jaeckel, J. Redondo and A. Ringwald, Phys. Rev. D 77, 095001 (2008); J. March-Russell, S. M. West, D. Cumberbatch and D. Hooper, JHEP 0807, 058 (2008); A. Ibarra, A. Ringwald and C. Weniger, JCAP 0901, 003 (2009); J. L. Feng and J. Kumar, Phys. Rev. Lett. 101, 231301 (2008).

[14] C. Coriano', N. Irges and E. Kiritsis, Nucl. Phys. B 746, 77 (2006); P. Anastasopoulos, F. Fucito, A. Lionetto, G. Pradisi, A. Racioppi, Y. S. Stanev, Phys. Rev. D 78, 085014 (2008); R. Armillis, C. Coriano and M. Guzzi, JHEP 0805, 015 (2008). 
[15] J. Kumar, A. Rajaraman and J. D. Wells, Phys. Rev. D 77, 066011 (2008); S. A. Abel, M. D. Goodsell, J. Jaeckel, V. V. Khoze, A. Ringwald, JHEP 0807, 124 (2008); C. P. Burgess, J. P. Conlon, L. Y. Hung, C. H. Kom, A. Maharana, F. Quevedo, JHEP 0807, 073 (2008).

[16] H. Georgi, Phys. Rev. Lett. 98, 221601 (2007); K. Cheung, W. Y. Keung and T. C. Yuan, Phys. Rev. Lett. 99, 051803 (2007).

[17] H. Goldberg and P. Nath, Phys. Rev. Lett. 100 (2008) 031803.

[18] P. Grajek, G. Kane, D. J. Phalen, A. Pierce and S. Watson, arXiv:0807.1508 [hep-ph]; V. Barger, W. Y. Keung, D. Marfatia and G. Shaughnessy, Phys. Lett. B 672, 141 (2009).

[19] C. R. Chen, F. Takahashi and T. T. Yanagida, Phys. Lett. B 671, 71 (2009); M. Pospelov and A. Ritz, Phys. Lett. B 671, 391 (2009); D. Hooper, P. Blasi and P. D. Serpico, JCAP 0901, 025 (2009); H. Yuksel, M. D. Kistler and T. Stanev, arXiv:0810.2784 [astro-ph]; I. Cholis, L. Goodenough and N. Weiner, arXiv:0802.2922 [astro-ph].

[20] T. Delahaye, R. Lineros, F. Donato, N. Fornengo and P. Salati, Phys. Rev. D 77, 063527 (2008); P. Brun, G. Bertone, J. Lavalle, P. Salati, R. Taillet, Phys. Rev. D 76, 083506 (2007).

[21] M. Cirelli, R. Franceschini and A. Strumia, Nucl. Phys. B 800, 204 (2008).

[22] M. Kamionkowski and S. M. Koushiappas, Phys. Rev. D 77, 103509 (2008).

[23] M. S. Longair, Cambridge, UK: Univ. Pr. (1994) 393 p

[24] D. Hooper and J. Silk, Phys. Rev. D 71, 083503 (2005).

[25] J. Hisano, S. Matsumoto, O. Saito and M. Senami, Phys. Rev. D 73 (2006) 055004.

[26] J. F. Navarro, C. S. Frenk and S. D. M. White, Astrophys. J. 490, 493 (1997); B. Moore, T. R. Quinn, F. Governato, J. Stadel, G. Lake, Mon. Not. Roy. Astron. Soc. 310, 1147 (1999).

[27] I. V. Moskalenko and A. W. Strong, Astrophys. J. 493, 694 (1998).

[28] M. Cirelli, M. Kadastik, M. Raidal and A. Strumia, arXiv:0809.2409 [hep-ph].

[29] E. A. Baltz and J. Edsjo, Phys. Rev. D 59, 023511 (1999).

[30] O. Adriani et al., Phys. Rev. Lett. 102, 051101 (2009).

[31] A. Bottino, C. Favero, N. Fornengo and G. Mignola, Astropart. Phys. 3, 77 (1995); L. Bergstrom, J. Edsjo and P. Ullio, Astrophys. J. 526, 215 (1999); T. Bringmann and P. Salati, Phys. Rev. D 75, 083006 (2007); A. Bottino, F. Donato, N. Fornengo and P. Salati, Phys. Rev. D 58, 123503 (1998); J. Lavalle, Q. Yuan, D. Maurin and X. J. Bi, arXiv:0709.3634,

[32] J. Chang et al., Nature 456, 362 (2008). 